\title{
An Iterative Weighted Reliability Decoding Algorithm for Two-Step Majority-Logic Decodable Cyclic Codes
}

\author{
Hsiu-Chi Chang, Chih-Lung Chen, and Hsie-Chia Chang
}

\begin{abstract}
An iterative weighted reliability two-step majority logic decoding (IWRTS-MLGD) algorithm for two-step majoritylogic (TS-MLG)-decodable cyclic codes is presented. In contrast to other message passing decoding algorithms that utilize real number operations, our proposed decoding algorithm requires only logical operations and integer additions. Therefore, large computational complexities can be reduced. For moderate-length TS-MLG-decodable cyclic codes, the proposed algorithm aided with soft information and a scaling factor outperforms the harddecision TS-MLGD algorithm and hard-decision BCH codes with similar length by $1.2-$ and $1.0-\mathrm{dB}$, respectively.
\end{abstract}

Index Terms-Finite geometry code, reliability-based message passing algorithm, two-fold EG code, cyclic code.

\section{INTRODUCTION}

$\mathbf{F}$ INITE geometry (FG) codes received great attention in the late 1960s and the early 1970s [1]-[3]. FG codes form a class of cyclic codes with reasonable minimum distance which can be decoded with simple majority logic (MLG). There are two types of MLG-decodable cyclic codes: onestep and multi-step. One-step MLG-decodable cyclic codes are rediscovered in [4] as FG-LDPC codes whose Tanner graphs are free of cycles of length 4. Long FG-LDPC codes decoded by the sum-product algorithm (SPA) [5] and minsum algorithm (MSA) [6] can nearly achieve Shannon's theoretical limit. However, multi-step MLG-decodable cyclic codes contain many short cycles of length 4 . Thus, standard SPA or MSA is not effective for decoding multi-step MLGdecodable cyclic codes [7] and [8]. In [7] and [8], a twostep soft decision decoding algorithm based on SPA and MSA was introduced for decoding TS-MLG-decodable cyclic codes, which is called the two-step iterative decoding algorithm (TSIDA). TS-IDA uses a five level message passing tree (MPT), which is constructed based on the orthogonal structure of the relation between frames and lines of the finite geometries. Therefore, it can avoid the degrading effects of short cycles. The simulation results in [8] show that TS-IDA outperforms other hard decision decoding methods by $2 \mathrm{~dB}$. However, the computational complexity is very large since a large portion of the computation involves real number.

Recently, [9] has introduced an efficient iterative algorithm called the soft reliability based iterative majority logic decoding algorithm (SRBI-MLGD) for decoding one-step

Manuscript received May 23, 2013. The associate editor coordinating the review of this letter and approving it for publication was G. Liva.

This work was supported by the NSC, Taiwan, R.O.C., under Contract NSC 101-2628-E-009-013-MY3.

The authors are with the Department of Electronics Engineering \& the Institute of Electronics, National Chiao Tung University, Hsinchu 30010, Taiwan, R.O.C. (email: jasper.ee94g@nctu.edu.tw, lung@si2lab.org, hcchang@mail.nctu.edu.tw).

Digital Object Identifier 10.1109/LCOMM.2013.082413.131191
MLG-decodable LDPC codes constructed by finite geometries. SRBI-MLGD is a binary message passing algorithm which utilizes only logical operations and integer additions. Thus, it greatly reduces the computational complexities compared to SPA or MSA. In contrast to other binary decoding methods such as differential binary message passing algorithm (DBMPA) [10], the SRBI-MLGD requires less computational complexity and memory.

In this letter, we propose an iterative weighted reliability two-step majority logic decoding (IWRTS-MLGD) algorithm for decoding TS-MLG-decodable cyclic codes. The idea of the algorithm is derived from SRBI-MLGD which greatly reduces the computational complexity compared to the previous TSIDA [8]. We use a subclass of TS-MLG-decodable cyclic FG codes called two-fold Euclidean geometry (EG) codes to demonstrate the effectiveness of the proposed algorithm. The letter is organized as follows: Section II introduces the background of the two-fold EG codes, and the corresponding two-step (TS)-MLGD algorithm. Section III provides the proposed IWRTS-MLGD algorithm in detail. The computational complexity analysis is also included. Section IV shows the simulation results. Finally, section V presents the conclusion.

\section{Construction of Two-Fold EG Codes And TS-MLGD ALGORITHM}

Consider a $d$-dimensional Euclidean geometry $\operatorname{EG}(d, q)$ over the field $\operatorname{GF}(q)$. The field $\operatorname{GF}\left(q^{d}\right)$ as an extension field of the field $\operatorname{GF}(q)$ is a realization of $\operatorname{EG}(d, q)$. Let $\alpha$ be a primitive element of $\operatorname{GF}\left(q^{d}\right)$. Then the powers of $\alpha, \alpha^{-\infty}=$ $0, \alpha^{0}=1, \alpha, \ldots \alpha^{q^{d}-2}$, represent the $q^{d}$ points of $\operatorname{EG}(d, q)$ and $\alpha^{-\infty}=0$ represents the origin of $\operatorname{EG}(d, q)$. Let $\mathrm{EG} *(d, q)$ be a subgeometry obtained from $\operatorname{EG}(d, q)$ by removing the origin and all the lines passing through the origin. Let $n=q^{d}-1$. There are $n$ nonorigin points and $J_{0}=n\left(q^{d-1}-1\right) /(q-1)$ lines not passing through origin in $\mathrm{EG} *(d, q)$. A line has $q$ points. If a point is on a line in $\mathrm{EG} *(d, q)$, we say that the line passes through the point(or orthogonal on the point). Every point in $\operatorname{EG} *(d, q)$ is intersected by $J_{1}=n /(q-1)-1$ lines. For the $i$-th line $L_{i}$ in $\mathrm{EG} *(d, q)$, where $0 \leq i<J_{0}$, there are $J_{2}=q^{d-1}-2$ lines parallel to it denoted as $L_{t, i}$, where $0 \leq t<J_{2}$. Let $\left\{L_{i}, L_{t, i}\right\}$ be a $(1,2)$-frame in $\mathrm{EG} *(d, q)$. There are $J_{2}(1,2)$-frames orthogonal on line $L_{i}$ denoted as $\left\{L_{i}, L_{0, i}\right\},\left\{L_{i}, L_{1, i}\right\}, \ldots,\left\{L_{i}, L_{J_{2}-1, i}\right\}$. There are a total of $r=n\left(q^{d-1}-1\right)\left(q^{d-1}-2\right) / 2(q-1)(1,2)$ frames in $\mathrm{EG} *(d, q)$. We consider an $r \times n$ matrix $\mathbf{H}$ over $\mathrm{GF}\left(q^{d}\right)$ with each row as a binary incidence vector of the $(1,2)$-frames in $\mathrm{EG} *(d, q)$. Then the null space of $\mathbf{H}$ gives a cyclic code of length $n$, called as two-fold EG [11] code. 
The generator polynomial $\mathbf{g}(X)$ of a two-fold EG code can be found in [8].

Let $\mathbf{u}=\left(u_{0}, u_{1}, u_{2}, \cdots, u_{n-1}\right)$ be a codeword of twofold EG code. Suppose $\mathbf{u}$ is transmitted via BPSK with unit energy over the binary AWGN with two-sided power spectral density $N_{0} / 2$. Let $\mathbf{y}=\left(y_{0}, y_{1}, y_{2}, \cdots, y_{n-1}\right)$ be the sequence of samples at the output of the sampler in the receiver. Let $\mathbf{z}=\left(z_{0}, z_{1}, z_{2}, \cdots, z_{n-1}\right)$ be the hard decision sequence of $\mathbf{y}$. The hard decision received sequence will be a two-fold EG codeword if $\mathbf{H z} \mathbf{z}^{T}=0$ or if the polynomial representation $\mathbf{z}(X)$ of $\mathbf{z}$ is divisible by the generator polynomial $\mathbf{g}(X)$. The paritycheck matrix $\mathbf{H}$ can be built by using the $(1,2)$-frames with $J_{0}$ lines in $\mathrm{EG} *(d, q)$. Consider the $i$-th line $L_{i}$ in $\mathrm{EG} *(d, q)$, where $0 \leq i<J_{0}$. Let $\mathbf{v}_{L_{i}}$ be the incidence vector of line $L_{i}$ denoted as $\mathbf{v}_{L_{i}}=\left\{v_{i, 0}, v_{i, 1}, \cdots, v_{i, n-1}\right\}$ whose components are the $n$-tuples over $\mathrm{GF}(2)$ that correspond to the $n$ nonorigin points of $\mathrm{EG} *(d, q)$ with $v_{i, j}=1$ if $\alpha^{j}$ is a point on $L_{i}$, otherwise $v_{i, j}=0$, where $0 \leq j<n$. Based on the $J_{0}$ lines in $\mathrm{EG} *(d, q)$, we form a $J_{0} \times n$ matrix denoted as $\mathbf{L}$, with $J_{0}$ incidence vectors of lines as rows and $n$ points as columns. Let $\mathbf{v}_{L_{0}}, \mathbf{v}_{L_{1}}, \cdots, \mathbf{v}_{L_{J_{0}-1}}$ be the rows of $\mathbf{L}$. For $0 \leq i<J_{0}$ and $0 \leq j<n$, we define $N_{i}=\left\{j: 0 \leq j<n, v_{i, j}=1\right\}$ and $M_{j}=\left\{i: 0 \leq i<J_{0}, v_{i, j}=1\right\}$. The indices in $N_{i}$ denote the location of 1-component in the $i$-th row of $\mathbf{L}$. The indices in $M_{j}$ denote the location of 1-component in the $j$-th column of L. Let $S\left(L_{i}\right)$ be the line-sum of $L_{i}$, which can be calculated by the inner product of $\mathbf{z}$ and the incidence vector $\mathbf{v}_{L_{i}}$ as follows:

$$
S\left(L_{i}\right)=\sum_{j \in N_{i}} z_{j}
$$

Consider a $(1,2)$-frame $F=\left\{L_{i}, L_{t, i}\right\}$ in $\mathrm{EG} *(d, q)$. The frame-sum of $F$ can be derived by XOR two line-sums: $S\left(L_{i}\right)$ and $S\left(L_{t, i}\right)$, denoted as $S(F)=S\left(L_{i}\right) \oplus S\left(L_{t, i}\right)$, where $\oplus$ is the XOR operation. Because $L_{t, i}$ is also a line in $\mathrm{EG} *(d, q)$, both $S\left(L_{i}\right)$ and $S\left(L_{t, i}\right)$ can be calculated by (1), which equal to either 0 or 1 . There are a total of $r$ frame-sums in the paritycheck matrix $\mathbf{H}$. Each frame-sum is the inner product of $\mathbf{z}$ and the binary incidence vector of $(1,2)$ frame $F$ composed of two lines $L_{i}$ and $L_{t, i}$ in $\mathrm{EG} *(d, q)$.

Consider updating a received bit $z_{j}$ in a two-fold EG code [11], where $0 \leq j<n$. The received bit $z_{j}$ corresponds to a point $\alpha^{j}$ in $\mathrm{EG} *(d, q)$. There are $J_{1}$ lines in $\mathrm{EG} *(d, q)$ passing through $\alpha^{j}$ denoted as $L_{u}^{j}$, where $0 \leq u<J_{1}$. The line-sum of $L_{u}^{j}$ is denoted as $S\left(L_{u}^{j}\right)$. These line-sums are orthogonal on $z_{j}$. For $0 \leq t<J_{2}$, there are $J_{2}$ lines in $\mathrm{EG} *(d, q)$ parallel to $L_{u}^{j}$, denoted as $L_{t, u}^{j}$. The line-sum of $L_{t, u}^{j}$ is denoted as $S\left(L_{t, u}^{j}\right)$. The first step of decoding is to decode $S\left(L_{u}^{j}\right)$ using the $J_{2}(1,2)$-frames of $\mathrm{EG} *(d, q)$ that are orthogonal on $L_{u}^{j}$. Let $F^{j, u, t}=\left\{L_{u}^{j}, L_{t, u}^{j}\right\}$ be a $(1,2)$-frame in $\mathrm{EG} *(d, q)$ that is orthogonal on $L_{u}^{j}$. The frame-sum of $F^{j, u, t}$ is denoted as $S\left(F^{j, u, t}\right)=S\left(L_{u}^{j}\right) \oplus S\left(L_{t, u}^{j}\right)$. The line-sum $S\left(L_{t, u}^{j}\right)$ of $S\left(F^{j, u, t}\right)$ is the extrinsic information for decoding $S\left(L_{u}^{j}\right)$. A received bit in $\mathbf{z}$ that is not contained in $L_{u}^{j}$ can appear in at most one $L_{t, u}^{j}$. With the above concept, we can decode the value of $S\left(L_{u}^{j}\right)$ correctly with MLGD using the $J_{2} S\left(F^{j, u, t}\right)$ that are orthogonal on $S\left(L_{u}^{j}\right)$ provided there are no more than $\left\lfloor J_{2} / 2\right\rfloor$ errors in $\mathbf{z}$. The second step of decoding is to decode $z_{j}$ by $J_{1} S\left(L_{u}^{j}\right)$ that are orthogonal on $z_{j}$. Any received bit of $\mathbf{z}$ other than $z_{j}$ can appear in at most one of these $J_{1}$ lines. These bits orthogonal on $z_{j}$ are the extrinsic information for $z_{j}$. The intrinsic information of $z_{j}$ comes from the hard-decision of itself. Since $J_{1}>J_{2}$, with MLGD based on these $J_{1}$ lines, the value of $z_{j}$ can be correctly decoded with no more than $\left\lfloor J_{2} / 2\right\rfloor$ errors in received bits [11]. The above decoding of a two-fold EG code is called two-step (TS)-MLGD.

\section{Iterative Weighted Reliability Decoding Algorithm FOR Two-Fold EG CODES}

TS-MLGD is a one-pass decoding algorithm with only hard-decision values from the received bits. TS-MLGD has low computational complexity, yet its performance can be greatly improved. In [8], TS-IDA algorithm improved the performance of two-fold EG codes by employing the soft information from the channel along with an iterative decoding process. The computational complexity of TS-IDA is very large because much of its computation involves real number. In the following, we propose an iterative decoding algorithm called iterative weighted reliability two-step-MLGD (IWRTSMLGD) algorithm which utilizes only logical operations and integer additions. The unweighted algorithm is also included.

Let $r_{j}$ be the quantized value of the sample $y_{j}$, where $0 \leq j<n$. The quantized value is an integer representation of the $2^{b}-1$ quantized intervals symmetric to the origin. Each interval has a length $\triangle$ and is represented by $b$ bits. Therefore, $r_{j}$ is in the range of $\left[-\left(2^{(b-1)}-1\right),+2^{(b-1)}-1\right]$. The magnitude $\left|r_{j}\right|$ of $r_{j}$ gives the soft measure of the reliability of the hard decision received bit $z_{j}$. Next, we need some notations to employ the iterative decoding process. Let $l$ be the iteration number. Let $l_{\max }$ be the maximum iterations to be performed in the decoding process. For $0 \leq l<l_{\text {max }}$, let $\mathbf{z}^{(l)}=\left\{z_{0}^{(l)}, z_{1}^{(l)}, \cdots, z_{n-1}^{(l)}\right\}$ be the received vector generated in the $l$-th iteration decoding, where $z_{j}^{(l)}$ is the $j$-th harddecision received bit at the $l$-th iteration. In each iteration, we first update the line-sum of all the lines by

$$
S^{(l)}\left(L_{i}\right)=\sum_{j \in N_{i}} z_{j}^{(l)} .
$$

Let $R_{j}^{(l)}$ be the reliability measure of the $j$-th bit at the $l$ th iteration. Moreover, let $\psi^{(l)}\left(L_{i}\right)$ be the reliability measure of $L_{i}$ at the $l$-th iteration, which is determined by the least reliable bit in $L_{i}$ as

$$
\psi^{(l)}\left(L_{i}\right)=\min _{j \in N_{i}}\left|R_{j}^{(l)}\right| .
$$

We set $R_{j}^{(0)}$ equal to the reliability $r_{j}$ as an initial reliability measure of a received bit $z_{j}$. Note that $S^{(l)}\left(L_{i}\right)$ and $\psi^{(l)}\left(L_{i}\right)$ can be shared during the decoding process for the received bits of $\mathbf{z}^{(l)}$ in an iteration. The received bit $z_{j}$ participates in $F^{j, u, t}$ consisting of two parallel lines, $L_{u}^{j}$ and $L_{t, u}^{j}$. The line-sum of $L_{u}^{j}$ and $L_{t, u}^{j}$ at the $l$-th iteration are denoted as $S^{(l)}\left(L_{u}^{j}\right)$ and $S^{(l)}\left(L_{t, u}^{j}\right)$, respectively. Both $S^{(l)}\left(L_{u}^{j}\right)$ and $S^{(l)}\left(L_{t, u}^{j}\right)$ can be derived from (2). Besides, $\psi^{(l)}\left(L_{t, u}^{j}\right)$ can be derived from (3). Suppose we update $z_{j}^{(l)}$ in $L_{u}^{j}$, where $0 \leq u<J_{1}$. The extrinsic information of $z_{j}^{(l)}$ comes from the following two 
steps. In the first step, the extrinsic information contributed by the $J_{2}$ parallel lines for the $u$-th line is calculated by

$$
\phi_{u}^{(l)}=\sum_{t=0}^{J_{2}-1} \psi^{(l)}\left(L_{t, u}^{j}\right)\left\{2 S^{(l)}\left(L_{t, u}^{j}\right)-1\right\} .
$$

Let $\varphi_{u}^{(l)}$ be the hard-decision of $\phi_{u}^{(l)}$, which can be expressed by an indicator function $\varphi_{u}^{(l)}=I\left(\phi_{u}^{(l)}>0\right)$. In the second step, the extrinsic information is contributed by the received bits of $\mathbf{z}^{(l)}$ that participate in $L_{u}^{j}$ except $z_{j}^{(l)}$ which is given by

$$
\sigma_{u, j}^{(l)}=S^{(l)}\left(L_{u}^{j}\right) \oplus z_{j}^{(l)} .
$$

The total extrinsic information can be derived from the aforementioned two steps by $J_{1} \sigma_{u, j}^{(l)} \operatorname{XOR} \varphi_{u}^{(l)}$ along with a scaling factor $\alpha$ as

$$
E_{j}^{(l)}=\alpha \sum_{u=0}^{J_{1}-1}\left\{2\left(\sigma_{u, j}^{(l)} \oplus \varphi_{u}^{(l)}\right)-1\right\},
$$

where $\alpha$ can be optimized by simulation. The reliability measure for the $j$-th received bit $z_{j}^{(l+1)}$ at the $(l+1)$-th iteration is calculated by

$$
R_{j}^{(l+1)}=R_{j}^{(l)}+E_{j}^{(l)} .
$$

In addition, in updating the soft reliability value $R_{j}^{(l)}$ for a received bit $z_{j}$, if the magnitude $\left|R_{j}^{(l)}+E_{j}^{(l)}\right|$ is larger than the quantization range $\left(2^{(b-1)}-1\right)$, the reliability value is set to $\left(2^{(b-1)}-1\right)$. Thus, the soft reliability value is always clipped under the maximal value of the quantization. If an unweighted decoding algorithm is desired, the scaling factor should be set to 1 . With the concept and notations defined above, the proposed algorithm is formulated as Algorithm 1.

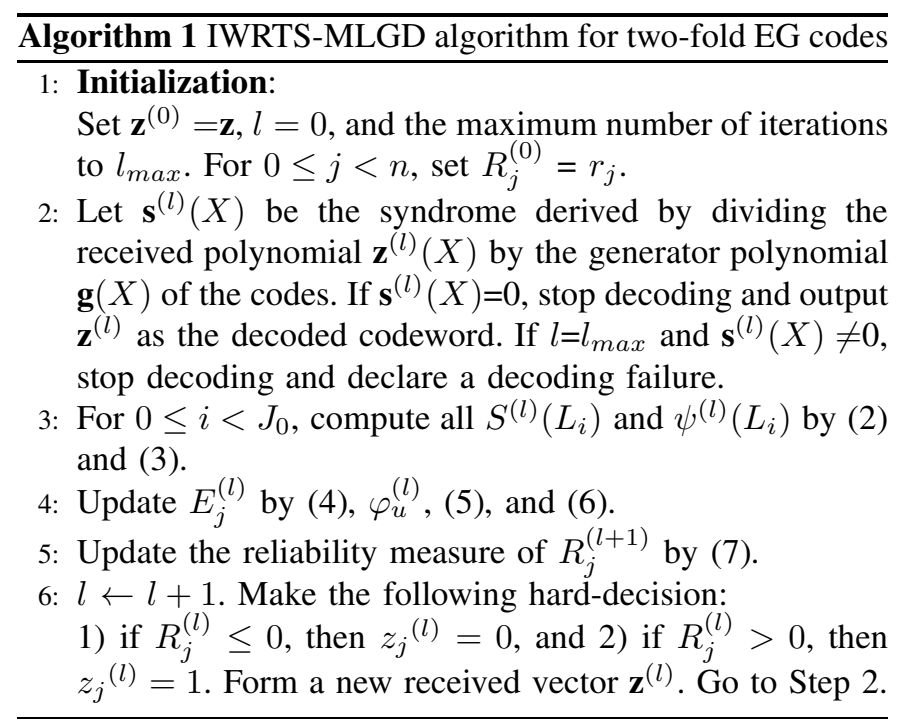

We analyze the computational complexity as follows. For step 3 , there are $J_{0}=n\left(q^{(d-1)}-1\right) /(q-1)$ lines in $\mathrm{EG} *(d, q)$. The line-sum of a line needs $(q-1)$ logical XOR operations. Thus, it needs $n\left(q^{(d-1)}-1\right)$ XOR operations to compute all the line-sums in $\mathrm{EG} *(d, q)$. In addition, it needs $q-1$ comparisons to determine the minimum value for the reliability of a line. Therefore, a total of $n\left(q^{(d-1)}-1\right)$
TABLE I

COMPUTATION COMPLEXITY REQUIRED PER ITERATION OF SPA, DBMPA, TS-IDA, AND PROPOSED IWRTS-MLGD ALGORITHM.

\begin{tabular}{c||c|c|c|c|c}
\hline \multirow{2}{*}{ Decoding algorithm } & \multicolumn{5}{c}{ Computation Cost per Iteration } \\
\cline { 2 - 6 } & BO & IA & RA & RM & Log \\
\hline SPA [5] & & & & $6 n^{2}$ & $\mathrm{n}$ \\
\hline DBMPA [10] & $4 n^{2}+n$ & $4 n^{2}+2 n$ & & & \\
\hline TS-IDA [8] & $3 n^{3 / 2}$ & & $n^{2}+n$ & & $7 n^{3 / 2}$ \\
\hline IWRTS-MLGD & $3 n^{3 / 2}$ & $n^{2}+n$ & & & \\
\hline \hline BO:Binary Operation; IA:Integer Addition; RA:Real Addition; \\
RM:Real Multiplication; Log:Logarithm;
\end{tabular}

comparisons is needed. In step $4, S^{(l)}\left(L_{u}^{j}\right)$ and $S^{(l)}\left(L_{t, u}^{j}\right)$ are derived by assigning their corresponding line-sums in $S^{(l)}\left(L_{i}\right)$. Meanwhile, $\psi^{(l)}\left(L_{t, u}^{j}\right)$ is derived by assigning its corresponding reliability of line in $\psi^{(l)}\left(L_{i}\right)$. In (4), it needs $J_{2}-1$ integer additions and $J_{2}$ logical operations for $\phi_{u}^{(l)}$. In addition, a logical operation is needed to determine the hard-decision of $\varphi_{u}^{(l)}$. (5) needs a logical operation and (6) needs $J_{1}-1$ integer additions with $J_{1}-1$ logical operations. The scaling factor $\alpha$ is set to be an integer for simplicity. If the scaling factor is an even number, the scaling can be accomplished with a simple shift operation. Otherwise, the scaling operation needs to add the original value to the shifted value, this will require another $J_{1} n$ integer additions. Step 5 requires $n$ integer additions to update the reliability of the $n$ received bits. Finally, step 6 needs $n$ logical operation to test the sign of an integer. To perform one iteration, we need $n\left(J_{1}+J_{2}+3\right)+J_{0}(q-1) \approx 3 q^{d-1}\left(q^{d}-1\right)$ logical operations, $J_{0}(q-1)+n\left\{J_{1} J_{2}-\left(J_{1}+J_{2}\right)+2\right\} \approx q^{d}\left(q^{d}-1\right)$ integer additions. Since the code length is $n=q^{d}-1$, by taking $d=2$ for two-fold EG codes constructed by two dimensional Euclidean geometry, the number of logical operations is of $O\left(n^{3 / 2}\right)$, while the number of integer additions is of $O\left(n^{2}\right)$.

In Table I we compare the numbers of operations per iteration required by SPA [5], DBMPA [10], TS-IDA [8], and the proposed IWRTS-MLGD. The numbers of operations for SPA and DBMPA are obtained from Table I in [9]. The number of operations for TS-IDA is derived from the analysis in [8]. In [9], $\delta$ is the number of 1-entries in the paritycheck matrix $\mathbf{H}$. There are $n\left(q^{d-1}-1\right)\left(q^{d-1}-2\right) / 2(q-1)$ rows in $\mathbf{H}$ with each row containing $2 q 1$ 's. Therefore, $\delta=2 q \times n\left(q^{d-1}-1\right)\left(q^{d-1}-2\right) / 2(q-1) \approx n^{2}$. With some translations, we demonstrate the complexity comparison in terms of $n$. Table I shows that the computation of the proposed IWRTS-MLGD with binary operations and integer additions employs the least operations compared to SPA and TS-IDA with real number operations. In addition, the proposed algorithm reduces computational complexity by at least 75 percent compared to DBMPA, which is also a binary message passing algorithm.

\section{Simulation Results}

In the following two examples, we use 8-bit uniform quantization with 255 levels and an interval length $\triangle=0.015$ for $(255,191)$ code, and 10-bit uniform quantization with 1024 levels and an interval length $\triangle=0.0075$ for $(1023,813)$ code. 


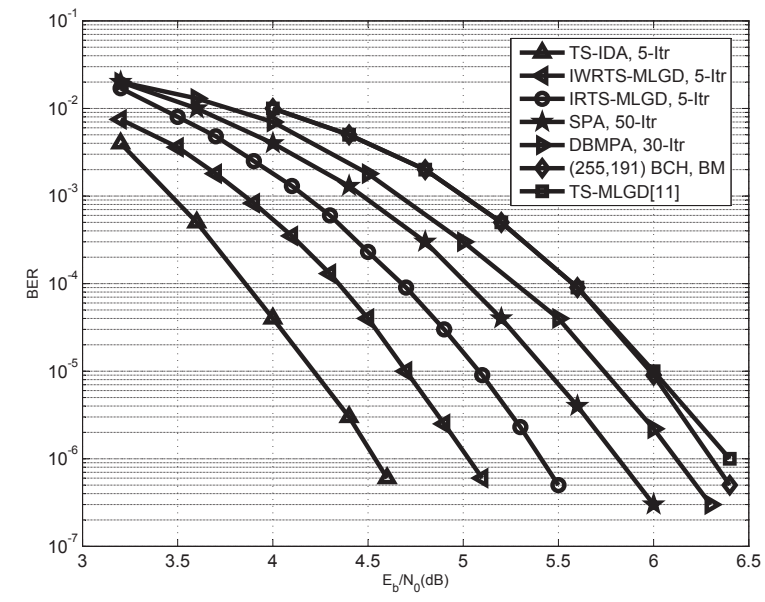

Fig. 1. Error performance of the two-fold EG code $(255,191)$ in Ex. 1 with various decoding algorithms over the AWGN channel.

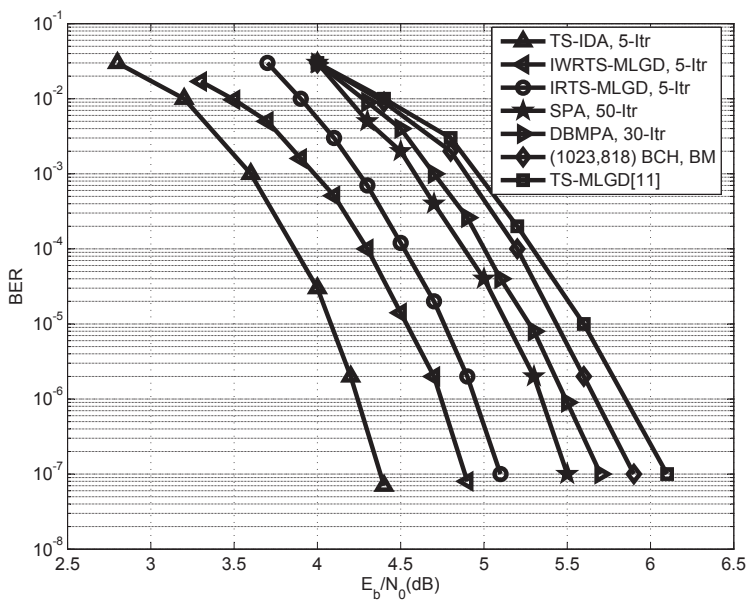

Fig. 2. Error performance of the two-fold EG code $(1023,813)$ in Ex. 2 with various decoding algorithms over the AWGN channel.

Both codes have scaling factor $\alpha=3$. The interval length and the scaling factor are obtained by simulation. The proposed unweighted decoding algorithm is denoted as IRTS-MLGD.

Example 1: Regarding $G F\left(2^{8}\right)$ as the geometry for the two-dimensional EG $\left(2,2^{4}\right)$, the two-fold EG code $(255,191)$ can be constructed. Fig. 1 shows the bit error performance of the proposed algorithms and TS-IDA with 5 iterations, DBMPA with 30 iterations, SPA with 50 iterations and TSMLGD. The performance of a $(255,191) \mathrm{BCH}$ code with minimum distance 17 decoded by the hard-decision BerlekampMassey(BM) algorithm [11] is also included. At BER of $10^{-6}$, the proposed IWRTS-MLGD degrades $0.5 \mathrm{~dB}$ from TS-IDA, but outperforms IRTS-MLGD, SPA, DBMPA, BCH code, and TS-MLGD by $0.4-, 0.8-, 1.2-, 1.3-$ and $1.4-\mathrm{dB}$, respectively.

Example 2: Regarding $G F\left(2^{10}\right)$ as the geometry for the two-dimensional EG $\left(2,2^{5}\right)$, the two-fold EG code $(1023,813)$ can be constructed. Fig. 2 depicts the bit error performance of the proposed algorithms, TS-IDA with 5 iterations, DBMPA with 30 iterations, SPA with 50 iterations and TS-MLGD. The performance of a $(1023,818) \mathrm{BCH}$ code with minimum distance 43 decoded by the hard-decision BM algorithm is also included. At BER of $10^{-7}$, IWRTS-MLGD degrades $0.5 \mathrm{~dB}$ from TS-IDA, but outperforms IRTS-MLGD, SPA, DBMPA, $\mathrm{BCH}$ code and TS-MLGD by 0.2-, 0.6-, 0.8-, 1- and 1.2-dB, respectively.

\section{CONCLUSION AND REMARKS}

In this letter, we develop an iterative weighted reliability decoding algorithm for TS-MLG-decodable cyclic codes that utilizes the orthogonal structure of the parity-check matrices. Although the algorithm is developed for a special subclass of TS-MLG-decodable cyclic codes, called two-fold EG codes, it can be applied to any TS-MLG-decodable cyclic code. Unlike TS-IDA using real number operations, our proposed algorithm utilizes only logical operations and integer additions, thus reducing computational complexity. From simulation results, though the two-fold EG codes decoded with the proposed IWRTS-MLGD algorithm are $0.5 \mathrm{~dB}$ away from TS-IDA, the proposed algorithm outperforms standard SPA and binary message passing DBMPA at least $0.6 \mathrm{~dB}$. Besides, it achieves $1 \mathrm{~dB}$ gain over similar length $\mathrm{BCH}$ codes decoded with the harddecision $\mathrm{BM}$ algorithm, and $1.2 \mathrm{~dB}$ gain over hard-decision TS-MLGD.

\section{REFERENCES}

[1] L. D. Rudolph, "Geometric configuration and majority logic decodable codes," M.E.E. thesis, University of Oklahoma, Norman, 1964.

[2] T. Kasami and S. Lin, "On majority-logic decoding for duals of primitive polynomial codes," IEEE Trans. Inf. Theory, vol. 17, no. 3, pp. 322-331, May 1968.

[3] S. Lin, "Multifold euclidean geometry codes," IEEE Trans. Inf. Theory, vol. 19, no. 4, pp. 537-548, July 1973.

[4] Y. Kou, S. Lin, and M. Fossorier, "Low-density parity-check codes based on finite geometries: a rediscovery and new results," IEEE Trans. Inf. Theory, vol. 47, pp. 2711-2736, Nov. 2001.

[5] F. R. Kschischang, B. J. Frey, and H. A. Loeliger, "Factor graphs and the sum-product algortihm," IEEE Trans. Inf. Theory, pp. 498-519, Feb. 2001.

[6] J. Chen and M. Fossorier, "Near optimum universal belief propagation based decoding of low density parity check code," IEEE Trans. Commun., pp. 406-414, Mar. 2002.

[7] L. Zhang, Q. Huang, and S. Lin, "Iterative decoding of a class of cyclic codes," in Proc. 2010 ITA.

[8] — - "Iterative algorithms for decoding a class of two-step majority logic decodable cyclic codes," IEEE Trans. Commun., vol. 59, no. 2, pp. 416-427, Feb. 2010.

[9] Q. Huang, J. K. Kang, L. Zhang, S. Lin, and K. Abdel-Ghaffar, "Two reliability-based iterative majority-logic decoding algorithms for LDPC codes," IEEE Trans. Commun., vol. 57, no. 12, pp. 3597-3606, Dec. 2009.

[10] N. Mobini, A. H. Banihashemi, and S. Hemati, "A differential binary message-passing LDPC decoder," IEEE Trans. Commun., vol. 57, no. 2, pp. 2518-2523, Feb. 2009.

[11] S. Lin and D. J. Costello, Jr., Error Control Coding: Fundamentals and Applications, 2nd ed. Prentice-Hall, 2004. 